\title{
ICER's assessment of lasmiditan, rimegepant, and ubrogepant for acute migraine
}

Meg Franklin, PharmD, PhD, and Eric Druyts, MSc

\section{Commentary}

Migraine is the third most prevalent and the sixth most disabling illness worldwide. ${ }^{1}$ Many patients who experience migraines suffer acute attacks a few times a month, but some patients report experiencing chronic daily $\mathrm{mi}^{-}$ graines, with at least 15 migraine days per month. Patients who experience migraines have a higher cost of care, decreased work productivity, and increased disability when compared with patients who do not experience migraines.

Treatment for migraine necessitates acute therapies to abort or control episodic symptoms. Treatments used for acute attacks are primarily triptans (5-hydroxytryptamine (5-HT) $1 \mathrm{~b} / 1 \mathrm{~d}$ receptor agonists), which were first introduced in the 1990s; there are currently 7 on the market. ${ }^{2}$ Although this class of medication has been shown to be safe and effective, there are patients who report that triptans do not provide adequate relief, lose efficacy over time, cause intolerable side effects, or are contraindicated. To circumvent these issues, patients are often reduced to taking opioids, which can result in misuse or medication overuse headaches. ${ }^{3}$

The U.S. Food and Drug Administration has recently approved 3 new drugs (lasmiditan, rimegepant, and ubrogepant) for the treatment of acute migraine in adults. Lasmiditan is a 5-HT1F receptor agonist, whereas rimegepant and ubrogepant act as calcitonin gene-related peptide (CGRP) antagonists. ${ }^{4,5}$ With the emergence of these new therapies, patients with migraine will have access to therapies in instances for which triptans are ineffective or are contraindicated because of cardiovascular risk. ${ }^{6}$ To assess the value of these new therapies, the Institute for Clinical and Economic Review (ICER) has published a report outlining its comparative effectiveness and cost-effectiveness.?

Two population models were considered in ICER's assessment. Population 1 included adults with moderate to severe migraine attacks who have not responded to nonprescription medicines and for whom triptans have not been effective, are not tolerated, or are contraindicated. Population 2 included only adults with migraine attacks who have not responded to nonprescription medicines and are eligible to use triptans. For population 1, the incremental cost-effectiveness ratio compared with standard of care for lasmiditan, rimegepant, and ubrogepant was $\$ 151,800$ per quality-adjusted lifeyear (QALY), $\$ 39,800$ per QALY, and $\$ 40,000$ per QALY, respectively. In population 2, lasmiditan, rimegepant, and ubrogepant were dominated by the 2 triptan therapies considered (sumatriptan and eletriptan). ${ }^{7}$

ICER's analysis acknowledges the benefits of these new treatments, and based on the analysis, rimegepant and ubrogepant are more cost-effective compared with lasmiditan in situations whereby triptans are not able to be effectively used. At the time the report was written, rimegepant pricing was not finalized and was assumed

\author{
Author affiliations \\ Meg Franklin, PharmD, PhD, Franklin \\ Pharmaceutical Consulting, Rock Hill, SC, \\ and Eric Druyts, MSc, Pharmalytics Group, \\ Vancouver, BC. \\ AUTHOR CORRESPONDENCE: \\ Meg Franklin, \\ mfranklin@franklin-pharma.com \\ J Manag Care Spec Pharm. \\ 2020;26(11):1464-66 \\ Copyright $\odot 2020$, Academy of Managed \\ Care Pharmacy. All rights reserved.
}

to be equal to ubrogepant, so results should be interpreted with caution. ICER's analysis was comprehensive and transparent; however, key areas require more thought and consideration. In this commentary, we discuss potential limitations and points for consideration for the ICER report on acute migraine.

\section{Real-World Evidence of Acute Migraine Treatments and Triptan Effectiveness}

The primary efficacy endpoint in the randomized clinical trials (RCTs) for the new therapies evaluated was freedom from pain at 2 hours after treatment. Pain relief (defined as a decrease 
in headache pain from moderate or severe at baseline to mild or no pain 2 hours after treatment and before rescue medication) was a secondary endpoint. While the assessment is limited by the available data for the newer therapies, there is some question as to how applicable the clinical trials on triptans are for comparison.

Treatment comparisons were made in the ICER report that compared modern-day RCTs with the triptan RCTs conducted 2 decades ago. Quality of RCTs and reporting have greatly improved from previous decades. In the newer trials, reporting of baseline patient characteristics, efficacy, and safety outcomes are more comprehensive. Examples include frequency of reporting of migraine attacks per month in the past 3 months and symptoms associated with migraine (nausea, vomiting, phonophobia, and photophobia). These parameters help determine disease severity to allow for appropriate direct and indirect comparisons between populations. Moreover, since then there has been an abundance of real-world evidence studies that have evaluated the effectiveness of triptans.

In addition, the discontinuation rates for lack of effectiveness used in the model for the triptans evaluated were assumed to be the same as that of the newer acute treatments for migraine (21.8\%). However, evidence suggests that triptan failure is common ( $\sim 50 \%)$, which is not addressed in the current ICER model. ${ }^{8-10}$ This presents an opportunity whereby the incorporation of real-world evidence can inform the model to ensure that longer-term, accurate data are used to generate results that are as relevant and meaningful as possible.

\section{Opioid Use in Patients with Migraines}

Observational studies also indicate that two thirds of individuals who do not respond to triptans receive an opioid prescription. ${ }^{11}$ The OVERCOME trial showed approximately $15 \%$ of individuals with 0-3 migraine headache days (MHDs) and $23.9 \%$ of individuals with $\geq 4$ MHDs per month used opioids..$^{12}$ Other studies have also reported similar rates of opioid use in migraine patients. ${ }^{9,13}$ Real-world evidence indicates that opioids are concomitantly or alternatively used with triptans. However, ICER did not account for opioid use in population 2 when comparing the new agents to triptans. Moreover, in both populations, potential opioid dependence and its associated adverse drug reactions were not reflected in ICER's cost-effectiveness model.

\section{Disutility Associated with Migraine Symptoms}

Approximately half of all acute migraine patients suffer from nausea, and $>90 \%$ suffer from phonophobia and photophobia. ${ }^{14,15}$ The report focuses on evaluating pain associated with acute migraine and did not incorporate disutility associated with other symptoms of the disease. Symptoms such as nausea and/or vomiting, photophobia, or phonophobia have been found to be debilitating in a high proportion of migraine patients. ${ }^{16-18}$ Nausea not only negatively affects an individual's quality of life, but can also cause a delay in the use and absorption of medication due to vomiting. ${ }^{19}$ Moreover, nausea/vomiting associated with migraine has been shown to worsen depression and lead to sleep disorders and impairment in activity. ${ }^{18}$ The ICER analysis limited comparators to oral triptans; therefore, disutility and lower response secondary to nausea/vomiting should also be taken into consideration. Migraine patients can also experience interictal photophobia, and these individuals have also been shown to have a greater risk for sleep disorders, depression, and anxiety. ${ }^{20}$

It was noted in ICER's response to comments that information pertaining to symptoms such as photophobia was not available in the clinical trials. For symptoms that affect such a large proportion of patients who suffer from migraines, we implore the drug manufacturers to incorporate this type of information into their trials and urge ICER to explore innovative ways to account for the effect of such symptoms.

\section{Indirect Costs}

Indirect costs were included as part of the scenario analyses, which ICER states is its customary approach, since models are developed from a payer perspective. However, the argument could be made that indirect costs should be included in the base-case analysis because indirect costs comprise a significant portion of total costs for patients with migraines..$^{21}$ Most patients with migraines are in the 18-44 age group and, therefore, are likely to be covered by insurance through their employers. ${ }^{22}$ Thus, capturing this information is not beyond the realm of capturing from a payer perspective. In fact, ICER did include indirect costs (specifically lost productivity) in a 2014 assessment on migraines. ${ }^{23}$

Also, the assumption of a quick treatment response (i.e., within 2 hours)-that patients would be able to begin, continue, or return to work-is likely to lead to an underestimation of true costs associated with total productivity loss. Many patients, even with current therapies, can be affected for several days per episode. ${ }^{24}$ 


\section{Conclusions}

Although there are limitations in ICER's analysis, its conclusion-that new therapies are viable alternatives for patients in which triptans are no longer effective, are not tolerated, or are contraindicated-is expected. Given the large number of patients who are in need of triptan alternatives, the availability of lasmiditan, rimegepant, and ubrogepant is timely and necessary for these patients to manage acute attacks, improve overall quality of life, and avoid potentially problematic treatments such as opioids. It should be reiterated that the results of the cost-effectiveness model were based on the assumption that pricing for rimegepant was not finalized and was assumed to be equal to ubrogepant. Now that rimegepant is available, results may be slightly different. In addition, as longer-term data become available, factors that affected model results (e.g., headache frequency and discontinuation rates) may affect the findings of the current assessment.

\section{DISCLOSURES}

No funding supported the writing of this commentary. The authors have nothing to disclose.

\section{REFERENCES}

1. Agosti R. Migraine burden of disease: from the patient's experience to a socioeconomic view. Headache. 2018;58 (Suppl 1):17-32.

2. Nicolas S, Nicolas D. Triptans. In: StatPearls. Updated May 26, 2020. StatPearls Publishing. Accessed October 1, 2020. https://www.ncbi.nlm.nih.gov/ books/NBK554507/

3. Thorlund K, Sun-Edelstein C, Druyts E, et al. Risk of medication overuse headache across classes of treatments for acute migraine. J Headache Pain. 2016;17(1):107.

4. Holland PR, Goadsby PJ. Targeted CGRP small molecule antagonists for acute migraine therapy. Neurotherapeutics. 2018;15(2):304-12.
5. Reuter U, Israel H, Neeb L. The pharmacological profile and clinical prospects of the oral 5-HT1F receptor agonist lasmidi$\tan$ in the acute treatment of migraine. Ther Adv Neurol Disord. 2015;8(1):46-54.

6. Do TP, Guo S, Ashina M. Therapeutic novelties in migraine: new drugs, new hope? J Headache Pain. 2019;20(1):37.

7. Atlas S, Touchette D, Agboola F, et al. Acute treatments for migraine: effectiveness and value. Final evidence report. Institute for Clinical and Economic Review. Updated May 14, 2020. Accessed October 1, 2020. https://icer-review. org/wp-content/uploads/2019/06/ ICER Acute-Migraine Final-EvidenceReport_updated_05142020.pdf

8. Lombard L, Farrar M, Ye W, et al. A global real-world assessment of the impact on health-related quality of life and work productivity of migraine in patients with insufficient versus good response to triptan medication. J Headache Pain. 2020;21(1):41.

9. Lipton RB, Marcus SC, Shewale AR, Dodick DW, Viswanathan HN, Doshi JA. Acute treatment patterns in patients with migraine newly initiating a triptan. Cephalalgia. 2020;40(5):437-47.

10. Deighton A, Johnston K, Dabirvaziri P, Harris L, L'Italien G, Lipton R. Switching and discontinuation patterns among triptan users: a systematic literature review. Neurology. 2020;94(15 Suppl):1929. Accessed October 1, 2020. https://n.neurology.org/content/94/15 Supplement/1929

11. Marcus SC, Shewale AR, Silberstein SD, et al. Comparison of healthcare resource utilization and costs among patients with migraine with potentially adequate and insufficient triptan response. Cephalalgia. 2020;40(7):639-49.

12. Ashina S, Foster SA, Nicholson RA, et al. OR10 Opioid use among people with migraine: results of the OVERCOME study. Headache. 2019;59(S1):11. Accessed October 1, 2020. https://headachejournal. onlinelibrary.wiley.com/doi/epdf/10.1111/ head.13549

13. Molina KC, Fairman KA, Sclar DA. Concomitant use of opioid medications with triptans or serotonergic antidepressants in US office-based physician visits. Drug Healthc Patient Saf. 2018;10:37-43.

14. Dodick DW. Migraine. Lancet. 2018;391(10127):1315-30.
15. Lipton RB, Buse DC, Saiers J, Fanning KM, Serrano D, Reed ML. Frequency and burden of headacherelated nausea: results from the American Migraine Prevalence and Prevention (AMPP) Study. Headache. 2013;53(1):93-103.

16. Ong JJY, De Felice M. Migraine treatment: current acute medications and their potential mechanisms of action. Neurotherapeutics. 2018;15(2):274-90.

17. Holroyd KA, Drew JB, Cottrell CK, Romanek KM, Heh V. Impaired functioning and quality of life in severe migraine: the role of catastrophizing and associated symptoms. Cephalalgia. 2007;27(10):1156-65

18. Gajria K, Lee LK, Flores NM, Aycardi E, Gandhi SK. Humanistic and economic burden of nausea and vomiting among migraine sufferers. J Pain Res. 2017;10:689-98.

19. Láinez MJ, García-Casado A, Gascón F. Optimal management of severe nausea and vomiting in migraine: improving patient outcomes. Patient Relat Outcome Meas. 2013;4:61-73.

20. Llop SM, Frandsen JE, Digre KB, et al. Increased prevalence of depression and anxiety in patients with migraine and interictal photophobia. J Headache Pain. 2016;17:34-34.

21. Polson M, Williams TD, Speicher LC, Mwamburi M, Staats PS, Tenaglia AT. Concomitant medical conditions and total cost of care in patients with migraine: a real-world claims analysis. Am J Manag Care. 2020;26(1 Suppl):S3-S7.

22. Burch R, Rizzoli P, Loder E. The prevalence and impact of migraine and severe headache in the United States: figures and trends from government health studies. Headache. 2018;58(4):496-505.

23. Institute for Clinical and Economic Review. Controversies in migraine management: a technology assessment.

August 19, 2014. Accessed October 1, 2020. https://icer-review.org/wp-content/ uploads/2016/01/CTAF_Migraine_Final_ Report_081914-2.pdf

24. Goadsby PJ, Holland PR, MartinsOliveira M, Hoffmann J, Schankin C, Akerman S. Pathophysiology of migraine: a disorder of sensory processing. Physiol Rev. 2017;97(2):553-622. 